\title{
Instrumentation numérique de la rédaction incrémentale : leçons tirées de la mise à l'épreuve du carnet numérique de l'élève chercheur
}

Matthieu Cisel

Institut des Humanités Numériques, CY Cergy Paris Université

Charlotte Barbier

Laboratoire Éducation Discours Apprentissages, Université de Paris

\section{Résumé}

Dans une perspective d'évaluation d'EIAH (environnements informatiques pour

l'apprentissage humain), nous étudions dans cette recherche les changements de pratiques d'enseignement liés à l'utilisation, en contexte scolaire, du CNEC (carnet numérique de l'élève chercheur), une application numérique instrumentant la rédaction incrémentale entre l'enseignant et les élèves. Au prisme du concept de contradiction développé dans la théorie de l'activité d'Engeström, nous cherchons à appréhender les difficultés associées à la rédaction incrémentale. Pour cela, nous avons mené des groupes de discussion et

Canadian Journal of Education / Revue canadienne de l'éducation 44:2 (2021)

(c)2021 Canadian Society for the Study of Education/ Société canadienne pour l'étude de l'éducation 
des observations en classe, avant et après l'introduction de l'application. Nos résultats corroborent l'hypothèse selon laquelle, dans un contexte de démarche d'investigation, l'instrumentation numérique de cette approche permet de mieux réguler l'activité des élèves que l'instrumentation papier. Par ailleurs, les entretiens collectifs révèlent que le rapport que les élèves entretiennent aux erreurs s'en trouve modifié, la correction de celles-ci étant moins considérée comme une sanction. La nécessité pour l'enseignant d'investir davantage de temps dans la formulation des retours ainsi que la multiplication des instruments, avec une redondance partielle entre instruments papier et instruments numériques, permettent de croire que de nouvelles contradictions pourraient émerger avec l'utilisation d'un tel EIAH. Mots-clés : rédaction incrémentale, EIAH, évaluation, numérique

\begin{abstract}
We studied how the introduction of the $C N E C$, a learning environment whose function is to foster iterative writing, affected the interactions between pupils and teachers in a sample of elementary and middle schools. Based on class observations and focus groups and through the lens of activity theory, we study the difficulties associated with the implementation of iterative writing, before and after the introduction of the CNEC. Our results corroborate the hypothesis that digital artifacts can improve the regulation of class activity in the context of inquiry-learning, compared to their paper equivalents. Parallelly, our results suggested that the perception students had of their own mistakes seems to change in a context of iterative writing, since the remarks of the teacher on such mistakes are less considered as a punishment. Observations also suggested that new contradictions could arise of the will of teachers to use both a paper-based and digital artifacts to perform iterative writing, and due to the increased time the formulation of feedbacks requires.
\end{abstract}

Keywords: iterative writing, evaluation, virtual learning environment 


\section{Introduction}

Après avoir défini la rédaction incrémentale et abordé la manière dont elle est traitée dans les travaux qui lui sont consacrés, nous reviendrons sur les angles morts que nous avons repérés dans la littérature scientifique. Nous nous pencherons ensuite sur le contexte de développement de l'environnement informatique pour l'apprentissage humain (EIAH) au centre de cette publication : le carnet numérique de l'élève chercheur (CNEC). Nous conclurons par une brève présentation du cadre théorique mobilisé dans cette recherche : la théorie de l'activité.

\section{Une problématique centrée sur la rédaction incrémentale et son instrumentation}

La rédaction incrémentale, parfois désignée par le vocable « écriture itérative » dans la littérature scientifique (Vardi, 2012), implique qu'une production écrite d'un élève, ou d'un groupe d'élèves, effectue des allers-retours entre les pairs et/ou l'enseignant. Elle est notamment utilisée dans les démarches d'investigation du fait de la nature cyclique et itérative que peut revêtir cette forme de démarche (Pedaste et al., 2015 ; Sandoval, 2004). Les travaux qui se sont penchés sur les retours, ou «feedbacks » dans la littérature anglosaxonne, ont permis de dresser des typologies de rétroactions (Hattie et Timperley, 2007 ; Kluger et DeNisi, 1996). Elles visent notamment à préciser dans quelles configurations celles-ci peuvent améliorer les écrits des élèves (ou étudiants) ou, au contraire, se révéler contreproductives.

Avec le développement de l'informatique, des programmeurs travaillent depuis des décennies à concevoir des artefacts numériques permettant d'instrumenter cette approche de la rédaction (Scardamalia, 2004) et, plus généralement, de faciliter la formulation de retours par l'enseignant (Bywater et al., 2019 ; Tabak et Reiser, 1997). Ainsi, nous disposons d'un certain nombre d'études de cas (Vardi, 2012 ; Zhao et Chan, 2014) centrées sur une instrumentation numérique de la rédaction incrémentale dans l'enseignement supérieur visant à démontrer les effets bénéfiques de l'introduction de l'artefact sur les productions écrites des élèves.

Quant aux recherches menées au niveau de l'enseignement primaire et secondaire, l'EIAH le plus emblématique des travaux sur la rédaction incrémentale est sans doute 
le Knowledge Forum (KF) (Scardamalia, 2004), fondé sur la théorie du Knowledge Building (Impedovo et Andreucci, 2016 ; Scardamalia et Bereiter, 2006). La littérature afférente détaille le fonctionnement des versions successives de l'outil (Hong et al., 2015 ; Laferrière et Lamon, 2010), mais reste centrée sur les collaborations entre élèves pour la reformulation des écrits, laissant de côté les interactions enseignants-élèves (Chan et Chan, 2011). Dans une revue de littérature portant sur ces interactions, Van de Pol et al. (2010) recensent les études montrant qu'au-delà de la question du caractère incrémental de la démarche, les retours formulés par les enseignants augmentent généralement les capacités d'autorégulation des élèves en plus de la qualité des écrits.

Néanmoins, la seule démonstration des apports potentiels de l'artefact pour les élèves ne suffit pas à favoriser son adoption. Pour ce faire, il est nécessaire de mieux comprendre l'appropriation qui en est faite selon le contexte d'utilisation, et les tensions que son utilisation peut engendrer ou, au contraire, alléger. En matière d'instrumentation numérique de la rédaction incrémentale, l'existence d'un angle mort dans la littérature sur les EIAH instrumentant la rédaction incrémentale nous a amenés à saisir l'opportunité qu'offrait le projet Savanturiers du Numérique pour développer une recherche sur le sujet.

Ce projet visait la mise au point du carnet numérique de l'élève chercheur $(C N E C)$, une application instrumentant diverses activités en classe, notamment la rédaction incrémentale. Dans le domaine de l'évaluation des EIAH, pour mieux comprendre comment un artefact peut être approprié, on se penche souvent sur son acceptabilité au prisme de l'utilité perçue de la technologie en question (Nielsen, 1993 ; Tricot et al., 2003). Pour ce faire, l'un des axes classiques de recherche consiste à se pencher sur la restructuration de l'activité qui accompagne l'utilisation de l'artefact, ainsi que sur les tensions afférentes ou, au contraire, qu'elle permet de dépasser. En nous appuyant sur une étude de cas, nous avons ainsi cherché à identifier les tensions associées à l'utilisation du CNEC, appréhendant celles-ci au prisme du concept de contradiction tel que développé dans la théorie de l'activité d'Engeström (1987). La question qui a guidé notre travail est la suivante : « Dans quelle mesure l'introduction d'un EIAH dédié à instrumenter la rédaction incrémentale contribue-t-elle à dépasser ou, au contraire, à renforcer les contradictions qui font obstacle à la mise en place de cette activité dans des projets en classe suivant une démarche d'investigation?» 
L'hypothèse principale de notre recherche était que les praticiens utilisant le CNEC allaient déplacer hors du temps de classe la rédaction des retours aux élèves, afin de faciliter la régulation des activités lors des séances. Ce déplacement rendrait alors l'utilisation de l'artefact plus acceptable pour les enseignants. L'hypothèse prend son origine dans des entretiens préliminaires avec des praticiens participant au programme Savanturiers, exerçant au collège, et qui avaient utilisé un logiciel de traitement de texte collaboratif pour instrumenter une rédaction incrémentale. Pour mettre à l'épreuve cette hypothèse, nous avons mené des observations en classe ainsi que des groupes de discussion avec les enseignants après avoir expérimenté l'utilisation du CNEC en classe. Après être revenus sur le contexte dans lequel a émergé l'application numérique, nous présenterons la théorie de l'activité qui a servi de cadre d'analyse à ce travail.

\section{Le contexte de développement du CNEC}

Le $C N E C$ comporte divers modules, dont plusieurs ont été pensés pour instrumenter une rédaction incrémentale de propositions à caractère scientifique (Cisel, Barbier et Baron, 2019). Ils sont utilisés au sein de projets relevant du programme Savanturiers. Ce programme éducatif français vise à développer, selon une logique de démarche d'investigation (Coquidé et al., 2009), des « projets de recherche » dans des classes du primaire et du secondaire, encadrés par des mentors généralement issus du milieu universitaire, afin d'initier les élèves aux méthodes de l'investigation scientifique (Cisel et al., 2019). Il promeut la liberté d'action des élèves, de la formulation de leur question de recherche à l'interprétation des résultats. Il vise plus généralement le renouvellement des approches pédagogiques dans l'enseignement des sciences, en mettant notamment l'accent sur le caractère itératif (Pedaste et al., 2015) de l'activité scientifique.

De 2016 à 2019, le programme Savanturiers a piloté un consortium nommé Les Savanturiers du Numérique dont l'objectif était de développer un artefact numérique destiné aux enseignants, visant notamment à instrumenter le caractère itératif de la démarche d'investigation. Ce consortium était composé des chercheurs de Savanturiers ; d'un industriel, Tralalère, qui a développé le code de l'artefact étudié ici et en est devenu propriétaire ; et du laboratoire de recherche EDA, qui a mené des recherches sur diverses questions en lien avec le projet. Le prototype de CNEC ainsi développé partage de nombreuses similitudes avec le Knowledge Forum (Scardamalia et Bereiter, 2013) 
ou le WISE (Slotta et Linn, 2009), deux environnements utilisés dans l'enseignement scientifique fondé sur l'investigation. Il s'inscrit dans la lignée de nombreux artefacts visant à instrumenter cette forme d'enseignement (Edelson, 2001 ; Linn et al., 2013 ; Quintana et al., 2004). Nous reviendrons plus en détail sur les modules au centre de cette contribution dans la section consacrée à la méthodologie.

Selon une logique de conception participative (Frascara, 2002), des enseignants des académies de Paris et de Créteil (France) exerçant l'enseignement primaire (6-11 ans) et secondaire (11-15 ans) ont intégré le consortium. Leur expérience des projets Savanturiers était variable : certains se lançaient pour la première fois, tandis que d'autres avaient déjà mené plusieurs projets auparavant. Tous avaient en commun le fait d'avoir déjà pratiqué la rédaction incrémentale avant l'utilisation du $C N E C$. Dès la fin de la première année de développement, tant les enseignants du primaire que ceux du collège ont pu mettre des prototypes à l'épreuve en classe (Cisel et al., 2019). En plus d'ouvrir leurs classes aux observations et de nous accorder des entretiens individuels, les enseignants ont été invités à participer à des groupes de discussion. Ces interactions ont constitué autant d'opportunités de collecter des données sur la question de la rédaction incrémentale.

\section{Une analyse menée au prisme de la théorie de l'activité}

La théorie de l'activité représente un cadre interdisciplinaire qui trouve ses origines dans les travaux en psychologie du développement, conduits dans les années 1930, en premier lieu par Vygotski. Au fil de ses formes successives, elle s'est développée pour devenir ce que l'on nomme parfois la théorie de l'activité de troisième génération (Engeström, 1987, 2000). Mobilisée par ses créateurs dans des contextes variés — du management (Engeström, 2011) aux transformations des établissements scolaires (Engeström, 2008), notre terrain d'étude — , elle vise à appréhender les actions individuelles ou collectives au prisme du système d'activité dans lequel elles s'inscrivent. Elle est largement utilisée dans le champ de l'éducation, et plus particulièrement dans le champ des technologies éducatives, de la formation à distance (Dir et Simonian, 2015), ou de la conception d'environnements informatiques (Bourguin, 2000 ; Bourguin et al., 2005). L'activité y est représentée par un modèle empirique constitué de six pôles en interaction : le sujet de 
l'activité, son objet, les règles qui la régissent, la communauté, la division du travail, et les instruments, ce dernier pôle constituant la focale de notre réflexion.

Selon Engeström (1987), tout système d'activité comporte des tensions internes qu'il nomme « contradictions ». Il en distingue plusieurs niveaux. Les contradictions primaires correspondent aux tensions au sein d'un pôle donné. Par exemple, dans le pôle « instruments », différents artefacts peuvent entrer en tension lorsqu'ils sont mobilisés de manière concomitante pour évaluer les compétences des élèves en matière d'acquisition de la démarche scientifique, notamment si l'enseignant utilise les cahiers individuels des élèves et un cahier rassemblant les productions de l'ensemble des membres d'un groupe. Les contradictions secondaires découlent des tensions entre deux pôles, tandis que les tertiaires correspondent aux tensions entre les anciens et les nouveaux éléments d'un pôle. Les quaternaires correspondent aux tensions entre les différents systèmes d'activité. Les systèmes d'activité sont dynamiques et évoluent lorsque les sujets tentent de dépasser les contradictions au sein de leur activité, par exemple en introduisant de nouveaux instruments. Les contradictions sont donc porteuses de transformations.

Nous plaçons l'enseignant au niveau du pôle « sujet », et définissons l'objet de l'activité comme le fait de mener un projet scientifique. Dans le pôle «communauté », nous incluons les élèves, le mentor du projet, les autres enseignants de l'établissement, les responsables du suivi au niveau de l'académie, et enfin les membres de l'équipe de Savanturiers avec lesquels le praticien est en contact. La « division du travail » porte sur le rôle des différentes parties prenantes dans les orientations impulsées au projet, même si les élèves disposent d'une certaine liberté d'action. Le programme n'attribue pas le choix des questions de recherche, des hypothèses et des protocoles à un seul des acteurs du projet (élève, enseignant, mentor), ceux-ci sont plutôt le fruit d'une interaction entre eux. Le pôle « instruments » comporte toutes les ressources mobilisées par les enseignants avec leurs élèves pendant le projet, ainsi que les ressources mises à disposition par les chercheurs de Savanturiers, tant pour le mentor que pour l'enseignant.

Enfin, le pôle « règles » inclut les règles mises en place par le ministère de l'Éducation nationale français, comme le respect des programmes officiels ; les règles plus ou moins explicitées du programme Savanturiers, telle la liberté d'action des élèves véhiculée par le vocable « élève chercheur »; et les règles locales de l'établissement et de l'enseignant. 
L'intérêt de la théorie de l'activité dans notre configuration est qu'elle permet d'appréhender simultanément, d'une part, les difficultés que rencontrent les praticiens avant l'introduction de la technologie et, d'autre part, l'éventuel dépassement de ces difficultés. La flexibilité qu'offre ce cadre théorique permet ainsi une plus grande opérationnalité dans le champ des technologies éducatives. Maintenant qu'ont été posées les bases de notre cadre théorique, revenons sur la méthodologie mise en œuvre.

\section{Méthodologie}

Dans cette partie, nous reviendrons sur les caractéristiques des modules du CNEC étudiés dans cet article. Puis, nous expliciterons les raisons de nos choix méthodologiques, qui seront ensuite développés dans deux sections dédiées aux modalités d'organisation des observations et des entretiens collectifs.

\section{La rédaction incrémentale au sein du $C N E C$}

Les chercheurs impliqués dans le projet ont contribué à la médiation entre les enseignants, les futurs utilisateurs potentiels et les programmeurs. L'un de leurs objectifs affichés, après des observations liminaires de projets, était d'instrumenter la rédaction incrémentale, notamment pour faire gagner du temps aux praticiens pendant le temps de classe. Deux modules du CNEC fonctionnent selon ce principe : la fiche-recherche (Figure 1) et le formulaire de séance. Seul le premier a effectivement été utilisé en classe par les enseignants sondés, c'est pourquoi nous nous concentrerons sur cette seule composante de l'artefact dans le cadre de cette contribution et ne traiterons pas du second module.

La fiche-recherche permet la production d'écrits structurés autour de cinq sections reflétant différentes étapes d'une démarche d'investigation (Pedaste et al., 2015) : question de recherche, hypothèse, protocole, données, conclusion (Figure 1) ${ }^{1}$. Elle a été pensée pour être utilisée de manière collective : la fiche est visualisable et modifiable

1 Des étayages (Bonnat, 2018) inspirés de travaux antérieurs ont également été conçus pour faciliter la rédaction d'écrits. Ils comprennent notamment des ouvertures de phrase et des questions d'auto-évaluation invitant l'élève à une certaine réflexivité sur son travail, avant l'envoi à l'enseignant; ils sont présentés dans le cadre d'un autre article. 
par tous les élèves d'un même groupe, constitué dans la plupart des classes par les enseignants et comportant deux à cinq élèves.

Il n'est pas obligatoire d'utiliser et de remplir chacune des sections. Ainsi, un groupe peut passer directement de la question au protocole si la question poursuivie n'appelle pas une démarche hypothético-déductive. Il est également possible de faire plusieurs propositions dans une même section, par exemple, en proposant plusieurs hypothèses pour une même question. Une fois que le groupe a rédigé une proposition pour une section donnée, l'enseignant peut laisser un commentaire sur celle-ci, et qualifier le travail des élèves en choisissant parmi trois options : « validé », «à corriger » ou « fermé ». Cette dernière option peut être utilisée pour signaler qu'il estime qu'il n'est pas pertinent de poursuivre cette piste. Les élèves voient un symbole de sablier affiché sur leur fiche-recherche tant que l'enseignant n'a pas traité la proposition ou un symbole indiquant que l'enseignant a revu la section. 


\section{Figure 1}

Captures d'écran de la fiche-recherche (haut) et de l'interface d'interaction permettant à l'enseignant de faire des commentaires sur les productions des élèves (bas)
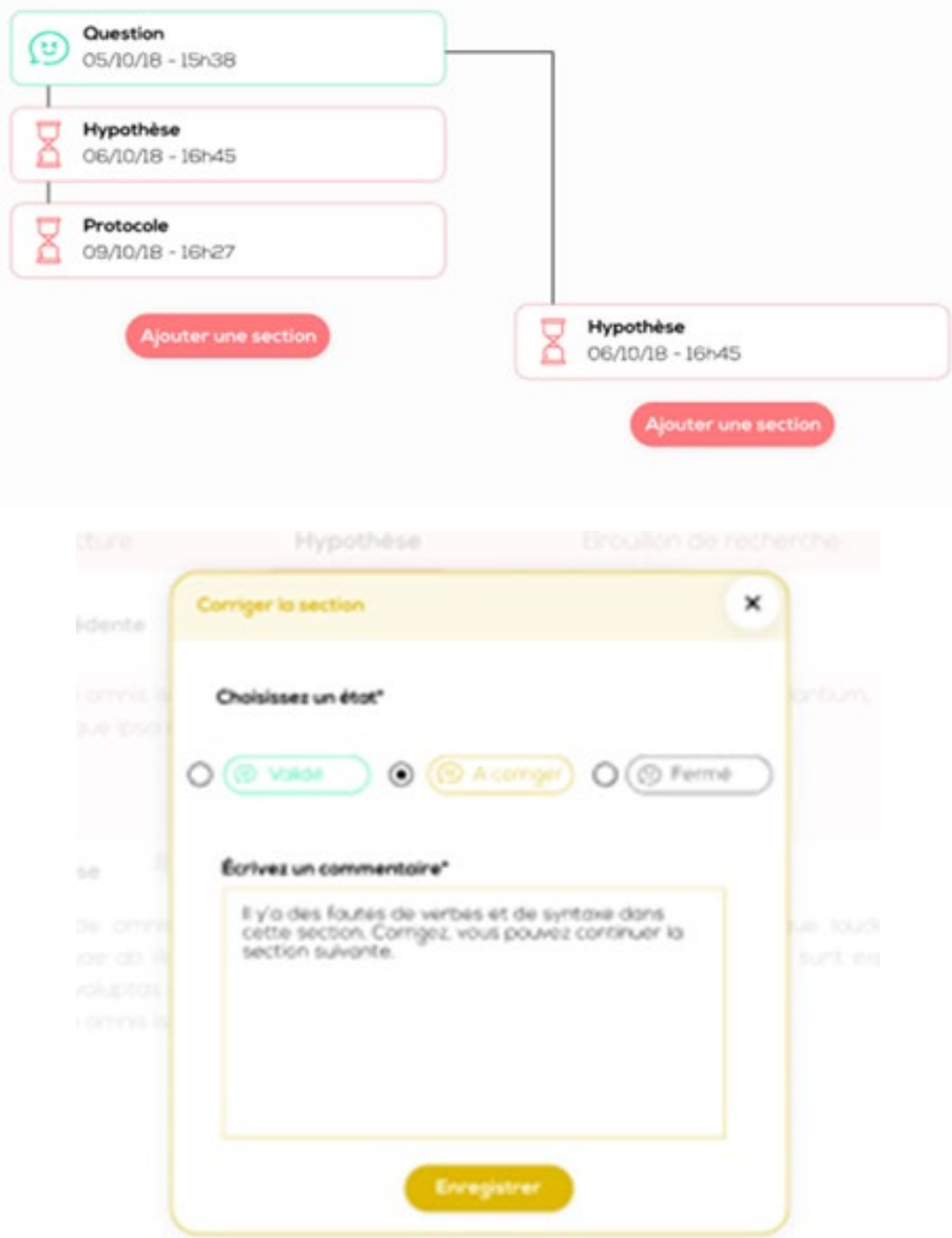


\section{La justification des choix méthodologiques}

Face au choix — récurrent dès lors que l'on doit évaluer un EIAH (Jamet, 2006 ; Nogry et al., 2004) - entre méthodes quantitatives et méthodes qualitatives, l'état d'avancement du $C N E C$ au moment de la recherche nous a amenés à préférer les secondes. Le caractère prototypique du $C N E C$ interférait avec la mise en place d'une expérimentation randomisée, car il devenait délicat de faire la distinction entre ce qui relevait de choix de conception et de bogues imprévus. Nous nous sommes donc appuyés sur les observations en classe et les entretiens (Nogry et al., 2004), plus en accord avec le cadre théorique de la théorie de l'activité, fondant nos analyses sur des notes prises au moment des observations. Nous avons préféré nous baser sur des notes plutôt que sur la captation vidéo des séances du fait des contraintes administratives et de la réticence des enseignants. Par ailleurs, ces méthodes, contrairement à des journaux de bord à remplir après chaque séance, avaient l'avantage de ne requérir que peu de temps de la part des praticiens, temps qui constituait une contrainte forte.

La possibilité de suivre des enseignants longitudinalement, sur plusieurs années, offrait l'opportunité de contraster leurs pratiques pédagogiques avant et pendant l'utilisation de l'application numérique. Cette configuration permet d'appréhender les évolutions des systèmes d'activité et des contradictions qui lui sont inhérentes ; la réalisation d'observations de la mise à l'épreuve de son utilisation en classe a dès lors été retenue. Pour Nogry et al. (2004), le point de vue de l'acteur semble préférable à tout autre quand il s'agit d'interpréter son activité : « Dans le cadre d'une évaluation en classe, il peut être utile de faire en complément des entretiens avec les enseignants pour confronter les différents points de vue sur le déroulement de l'évaluation » (p. 268).

Comme le souligne Jamet (2006) dans sa réflexion sur les protocoles verbaux, « [c]es verbalisations peuvent être concurrentes à la tâche mais elles ralentissent le traitement. Elles peuvent être différées après la tâche, par exemple en diffusant la vidéo du protocole au sujet et en lui demandant de la commenter » (p. 142). Cependant, comme souligné plus tôt, nous n'avions pas les autorisations nécessaires pour réaliser de tels enregistrements, et nous avons préféré échanger en aval des séances sur les choix pédagogiques effectués, via des entretiens individuels, non présentés ici, ou de groupe. En amont de leur mise en œuvre, les guides d'entretien contenant les questions posées lors des groupes de discussion et les grilles d'observation ont été confrontés au regard d'un 
chercheur en sciences de l'éducation, ainsi qu'à une ancienne enseignante du programme Savanturiers, afin de mettre à l'épreuve la pertinence de nos instruments de recherche. Nous donnons plus de détails sur leur construction dans les paragraphes qui suivent.

\section{Les observations en classe}

Nous avons suivi de manière longitudinale les projets de sept classes différentes. Sur ces sept projets, quatre ont mis en place une rédaction incrémentale des différentes productions scientifiques (questions, hypothèses, etc.), nous permettant ainsi de contraster les observations de manière qualitative avant et après l'introduction du CNEC. Avant chaque utilisation de l'artefact, dans les jours précédant l'observation, les enseignants nous informaient de la manière dont ils envisageaient de scénariser la séance, sans que nous cherchions à influer sur le scénario. Chaque groupe d'élèves disposait d'au moins une tablette ou un ordinateur, et un élève du groupe était désigné comme secrétaire pour entrer les éléments exigés par l'enseignant dans l'application.

Des notes étaient prises de manière synchrone dans un premier temps, puis des comptes-rendus plus structurés étaient produits sur la base de ces notes. Les observations ont porté sur le déroulement des activités mises en place par l'enseignant, en nous concentrant sur l'activité des enseignants, les suivant, notamment, lorsqu'ils passaient d'un groupe à l'autre. Nous avons mobilisé une grille d'analyse reprenant les six pôles du modèle d'Engeström pour structurer nos comptes-rendus, ainsi que les contradictions qui reliaient ces différents pôles pour catégoriser les difficultés rencontrées tant par les enseignants que par les élèves. Cette approche conduit potentiellement à une collecte volumineuse de données. Pour pallier ce problème, nous nous sommes concentrés, au cours des observations, sur les difficultés en lien avec un nombre limité d'axes de recherche, comme l'évaluation des compétences scolaires (Cisel et Baron, 2019) et la rédaction incrémentale, focale de la présente contribution.

Le Tableau 1 recense les caractéristiques des quatre projets ayant utilisé une rédaction incrémentale, la période d'observation considérée, ainsi que le nombre de séances observées. Le nom des établissements a été modifié pour anonymiser l'étude ; il en va de même pour le nom des enseignants. 


\section{Tableau 1}

Détail des observations menées en classe, avec des projets non instrumentés par le CNEC (2016-2017), puis avec des projets instrumentés (2017-2019)

\begin{tabular}{lcccc}
\hline \multicolumn{1}{c}{ Code étab. } & Niveau & Académie & \# Enseignants & \# séances observées \\
\hline Bouliers & Élémentaire (CE2) & Paris & 2 & $8(2017-2019)$ \\
Saint-Victor & Collège (6e) & Paris & 2 & $9(2016-2017)$ \\
André Girault & Collège (5e) & Paris & 3 & $9(2016-2017)$ \\
Peupliers & Élémentaire (CM2) & Créteil & 1 & $8(2016-2017)$ \\
\hline
\end{tabular}

\section{Les modalités d'organisation des groupes de discussion}

Dix-huit comités de conception réunissant les membres du consortium et des enseignants partenaires ont été organisés sur la période de 2016-2019, dont huit ont donné lieu à des groupes de discussion (Cisel et al., 2019). Ces rencontres se sont étalées sur les trois années qu'a duré le consortium, en premier lieu, au moment de la constitution du cahier des charges, pour permettre aux praticiens d'exprimer leurs besoins. Chaque séance de discussion en groupe (Krueger, 2014), d'une durée d'une heure environ, réunissait entre quatre et huit enseignants, selon leur disponibilité. Les premières étaient centrées sur les maquettes du $C N E C$, avant son développement (Cisel et Baron, 2019). Au fur et à mesure de l'avancement du projet, les comités se sont concentrés sur la prise en main de l'application, c'est-à-dire sur la connaissance de ses fonctionnalités et des modalités de son utilisation en classe. Les derniers comités ont été consacrés aux retours des enseignants faisant suite à la mise à l'épreuve de l'application en classe.

Un chercheur a mené l'ensemble des groupes de discussion en se basant chaque fois sur un guide d'entretien élaboré à partir de nos questions de recherche et des données déjà recueillies. Le guide était spécifique à chaque rencontre et aux thèmes abordés dans la discussion. Les discussions de groupe étaient enregistrées puis transcrites dans leur intégralité sous forme de fichiers texte. Elles étaient ensuite analysées avec une grille thématique fondée sur les six pôles de la théorie de l'activité, grille visant à faire apparaître les contradictions entre les pôles. La rédaction incrémentale a fait l'objet d'une séquence dédiée à la fin de la troisième année du projet de recherche. Les questions étaient fondées sur les observations réalisées en classe en amont, l'objet des rencontres 
étant de compléter les observations et d'éclairer les changements de pratiques que nous avions constatés. Les questions traitées lors de la séance consacrée à la rédaction incrémentale peuvent être regroupées en deux thématiques. La première correspond aux changements dans la manière de pratiquer la rédaction incrémentale à la suite de l'introduction du $C N E C$, pendant les séances et en dehors du temps de classe, une focale étant portée sur les difficultés correspondantes. La seconde correspond à la perception par les élèves de ces changements de pratiques. Les enseignants ayant participé à ce groupe de discussion avaient tous utilisé la fiche-recherche pendant plusieurs semaines dans le cadre de leur projet et les verbatim présents dans cet article en sont issus.

\section{Résultats}

La présentation des résultats est structurée en deux temps. Dans un premier temps, nous revenons sur une série de contradictions qui traversaient le système d'activité des enseignants, et que l'introduction de la fiche-recherche visait à dépasser. Dans la mesure où l'ensemble des enseignants mettaient en place cette approche avant de rejoindre le projet de recherche Les Savanturiers du Numérique, la réflexion est centrée sur l'introduction d'un instrument numérique, et non sur celle de la rédaction incrémentale per se. L'hypothèse d'un dépassement partiel des contradictions, permis par une rédaction des retours par l'enseignant en dehors du temps de classe, semble corroborée par nos observations. Cette restructuration conduit à une réorganisation de l'activité qui dépend du niveau d'enseignement. Dans un second temps, nous revenons sur les données recueillies lors des discussions en groupe, nous concentrant sur les perspectives des enseignants vis-à-vis de l'utilisation de ce module du CNEC. Au-delà de la question de la restructuration de l'activité, une nouvelle hypothèse émerge : avec l'instrumentation numérique, les élèves seraient plus aptes à prendre en compte les retours reçus par écrit lors de la reformulation de leurs propositions. Pour les praticiens sondés, ceci tiendrait en partie du fait que le rapport à l'erreur est sensiblement modifié lorsque la rédaction incrémentale est instrumentée numériquement. 


\section{L'introduction du CNEC et la réorganisation de l'activité : une analyse des observations en classe}

Des tensions liées à la mise en place d'une rédaction incrémentale ont été identifiées lors des observations en classe précédant l'introduction de l'application numérique. Nous revenons sur quatre d'entre elles (Tableau 2) qui constituent autant de contradictions au prisme de la théorie de l'activité. Dans le Tableau 2, nous faisons correspondre les contradictions repérées aux modalités de leur dépassement partiel au moment de l'introduction du CNEC. Néanmoins, des tensions nouvelles apparaissent en lien à la restructuration de l'activité induite par l'utilisation du $C N E C$.

Les difficultés repérées avant l'introduction du CNEC. Il nous faut revenir en premier lieu sur les contradictions primaires et secondaires traversant le système d'activité des enseignants avant même l'introduction du CNEC. Les observations nous permettent d'identifier au moins quatre difficultés qui relèvent tantôt de contradictions primaires au sein du pôle « règles », tantôt de contradictions secondaires entre les pôles « règles » et « instruments ».

Au sein du pôle « règles », nous constatons une tension entre les règles du programme Savanturiers (Figure 2, flèche bleue), qui préconise des démarches d'investigation laissant beaucoup de place à l'agentivité de l'élève, et les règles du ministère de l'Éducation nationale français, notamment la contrainte du respect des programmes. Toute activité en classe conduisant à renoncer à une partie significative des programmes en raison du temps nécessaire pour la mettre en œuvre mène à des tensions au sein du système d'activité des enseignants. La difficulté à mettre en place la rédaction incrémentale dans les projets ne constitue qu'une déclinaison parmi d'autres de cette contradiction primaire initiale. En effet, celle-ci implique, par définition, de faire des allers-retours parfois nombreux entre l'enseignant et les élèves.

Tout au long du projet, de la rédaction des questions de recherche à celle des conclusions, les élèves doivent eux-mêmes reformuler leurs productions, ce qui peut occasionner des délais considérables entre la première et la dernière formulation d'un écrit si l'enseignant respecte l'agentivité des élèves. Ainsi, dans un cas extrême, celui du collège Saint-Victor, il n'a pas fallu moins de 4 séances de 90 minutes pour que tous les groupes aient proposé une formulation de question jugée satisfaisante par l'enseignant avant l'introduction du $C N E C$. Sur les 14 séances consacrées au projet 
Savanturiers, certains groupes ont consacré 8 séances, soit plus de la moitié des cours de cette année scolaire, à la reformulation de productions écrites, questions, hypothèses, ou protocoles. Cette manière de procéder entre en contradiction avec la règle du respect des programmes, car elle a imposé à l'enseignant de renoncer à traiter certains points obligatoires du programme, ou à les traiter de manière superficielle, selon sa propre analyse.

\section{Tableau 2}

Tensions recensées au moment des observations, et changements de pratiques à la suite de l'introduction du CNEC

\begin{tabular}{|c|c|c|c|}
\hline $\begin{array}{l}\text { Tensions préexistantes à l'intro- } \\
\text { duction du CNEC }\end{array}$ & $\begin{array}{c}\text { Étab. } \\
\text { concernés }\end{array}$ & $\begin{array}{c}\text { Changements de pratiques } \\
\text { après l'introduction du CNEC }\end{array}$ & $\begin{array}{c}\text { Étab. } \\
\text { concernés }\end{array}$ \\
\hline $\begin{array}{l}\text { Reste de la classe fonctionnant } \\
\text { au ralenti lorsque l'enseignant } \\
\text { se concentre sur le travail de } \\
\text { rédaction d'un groupe en par- } \\
\text { ticulier }\end{array}$ & $\begin{array}{l}\text { Bouliers, } \\
\text { Saint-Victor, } \\
\text { Peupliers }\end{array}$ & $\begin{array}{l}\text { Rédaction en dehors du temps de } \\
\text { classe des retours sur la formula- } \\
\text { tion des productions, concentra- } \\
\text { tion en présentiel sur l'avance- } \\
\text { ment des projets, plus grande } \\
\text { surveillance de la classe }\end{array}$ & $\begin{array}{l}\text { Bouliers, } \\
\text { Saint-Victor, } \\
\text { Peupliers }\end{array}$ \\
\hline $\begin{array}{l}\text { Répétitions des mêmes retours } \\
\text { d'une séance à l'autre }\end{array}$ & $\begin{array}{l}\text { Saint-Victor, } \\
\text { Bouliers }\end{array}$ & $\begin{array}{l}\text { Renvoi des élèves sollicitant } \\
\text { l'enseignant aux retours réalisés à } \\
\text { l'écrit en lieu et place de nouvelles } \\
\text { explications faites à l'oral }\end{array}$ & Bouliers \\
\hline $\begin{array}{l}\text { Difficulté à gérer, sur la durée } \\
\text { de la séance, la multiplication } \\
\text { des sollicitations de l'ensemble } \\
\text { des groupes vis-à-vis de leurs } \\
\text { productions }\end{array}$ & $\begin{array}{l}\text { Bouliers, } \\
\text { Saint-Victor, } \\
\text { André Girault }\end{array}$ & $\begin{array}{l}\text { Renvoi des élèves sollicitant } \\
\text { l'enseignant aux retours réalisés à } \\
\text { l'écrit }\end{array}$ & $\begin{array}{l}\text { Bouliers, } \\
\text { Saint-Victor }\end{array}$ \\
\hline $\begin{array}{l}\text { Difficultés d'accès aux cahiers } \\
\text { en dehors des séances de classe } \\
\text { compliquant la rédaction de } \\
\text { retours en asynchrone }\end{array}$ & $\begin{array}{l}\text { Bouliers, } \\
\text { André Girault, } \\
\text { Peupliers, } \\
\text { Saint-Victor }\end{array}$ & $\begin{array}{l}\text { Difficulté d'accès aux produc- } \\
\text { tions des élèves sur le } C N E C \text { en } \\
\text { cours de séance conduisant à une } \\
\text { utilisation simultanée du cahier et } \\
\text { de l'application pour le suivi des } \\
\text { productions }\end{array}$ & $\begin{array}{l}\text { Bouliers, } \\
\text { André Girault, } \\
\text { Peupliers, } \\
\text { Saint-Victor }\end{array}$ \\
\hline
\end{tabular}




\section{Figure 2}

Une modélisation des contradictions traversant le système d'activité « Projet Savanturiers non instrumenté par le CNEC », selon le modèle d'Engeström (1987)

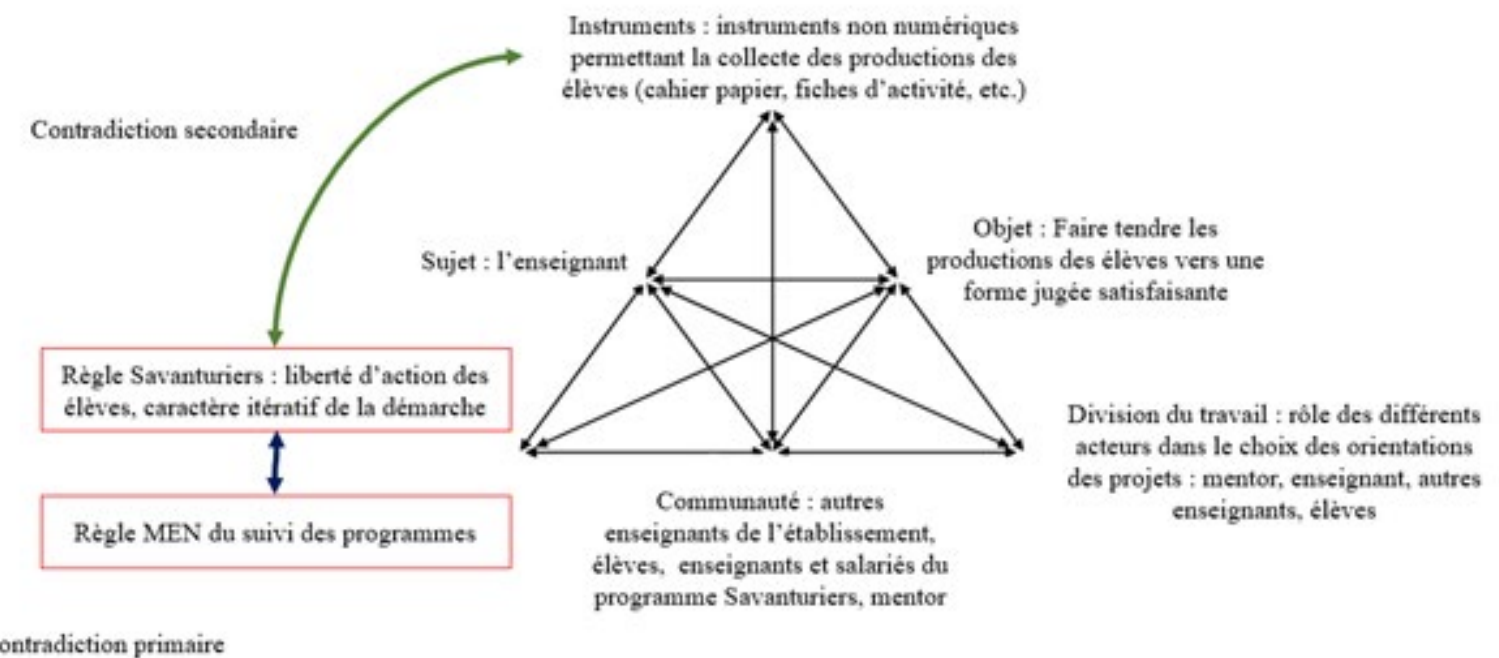

L'hétérogénéité des vitesses d'avancement tient largement au fait que lorsque l'enseignant se concentre sur un groupe, les autres élèves sont pour la plupart désinvestis. Le plus souvent, les élèves ne prennent pas de notes des remarques orales de l'enseignant, qui lui ne les écrit pas systématiquement sur leurs cahiers. Les commentaires effectués semblent oubliés rapidement après le passage de l'enseignant. Dès lors, l'enseignant, de séance en séance, refait généralement les mêmes remarques lorsqu'il se penche sur les productions d'un groupe, accusant ainsi une perte de temps.

Les tensions générées par le caractère chronophage du travail de reformulation sont soulignées en particulier par les enseignants plus dirigistes, qui priorisent la contrainte des programmes aux règles du programme Savanturiers. Au collège André Girault, trois séances sont consacrées à la formulation de la version définitive de la question de recherche, là où les enseignants estiment que la formulation de la question pourrait être réalisée en une seule séance, voire ne pas être faite par les élèves mais seulement par les enseignants.

Lorsque les élèves sont proactifs et formulent de nombreuses questions, ce qui a par exemple été observé dans l'école primaire Bouliers, une contradiction secondaire 
entre le pôle « règles » et le pôle « instruments » apparaît (Figure 2, flèche verte). Les enseignantes sont sollicitées de manière répétée au cours de chaque séance par des élèves qui viennent avec leur cahier pour demander des retours à chaque nouvelle itération d'une production écrite. Dans la mesure où les praticiens ne sont pas en mesure d'emporter les cahiers à la maison après chaque séance, pour lire chez eux les productions des élèves, la nécessité de répondre aux sollicitations de l'ensemble de la classe interfère avec leur activité de suivi individuel durant laquelle ils passent d'un groupe à l'autre pour s'assurer du bon déroulement de chaque projet. La nécessité d'utiliser en cours de séance les cahiers papier comme support de la rédaction incrémentale engendre une contradiction qui sera en partie dépassée au moment de l'introduction du CNEC. Cette considération nous amène à la question des changements de pratiques qui ont découlé spécifiquement du caractère numérique de l'artefact.

Les changements de pratiques à la suite de l'introduction du CNEC. Si la contradiction primaire au sein du pôle « règles » demeure - elle est inhérente aux objectifs des projets Savanturiers — , la contradiction secondaire entre les pôles « règles » et « instruments » semble partiellement dépassée au moment de l'introduction du CNEC. Sans se concerter, ni avec les collègues ni avec l'équipe de conception, quant à la manière d'utiliser la fiche-recherche, les pratiques des praticiens semblent converger sur un certain nombre de points lors des tests du module en classe. En premier lieu, pour trois des quatre établissements suivis (Tableau 2), les enseignants décident d'effectuer hors du temps de classe leurs retours sur les productions écrites des élèves. S'ils continuent à passer de groupe en groupe, ils passent moins de temps à échanger à l'oral avec certains groupes, en particulier sur des questions de formulation, et davantage de temps à s'assurer que l'ensemble des élèves sont actifs et font des progrès à chaque séance. Alors que sur certaines séances, dans plusieurs classes (Saint-Victor, Bouliers), nous observions avant l'introduction du CNEC que certains groupes pouvaient mobiliser le tiers du temps d'un enseignant pendant la séance, ce type de dynamique s'estompe après l'introduction de l'artefact. À Bouliers, par exemple, les enseignantes, lorsqu'elles constatent une faible évolution des productions d'une séance sur l'autre, finissent par refuser de faire à l'oral des retours qu'elles avaient déjà faits auparavant; elles renvoient systématiquement les élèves aux retours qu'elles ont faits par écrit, pour rechercher des gains de temps. 
Il en va de même, notamment dans cette école, pour la gestion des sollicitations répétées en cours de séance pour des corrections. Les élèves qui viennent voir l'enseignante sont également renvoyés à leur place, avec pour instruction de se concentrer sur d'autres activités que la question de formulation et d'attendre que des retours soient formulés à l'écrit.

Si ces évolutions semblent relever d'un dépassement de la contradiction secondaire identifiée plus tôt, puisqu'elles permettent une meilleure gestion du temps de classe, une nouvelle tension entre les pôles « règles » et « instruments » semble émerger. Nous n'observons pas un remplacement du cahier papier par le $C N E C$, mais une utilisation des deux supports par l'ensemble des enseignants étudiés. Ils demandent alors aux élèves de rédiger deux fois leurs productions : sur l'application numérique et sur le cahier de groupe papier. Le premier est utilisé pour faire des retours détaillés, hors du temps de classe, le second pour suivre brièvement l'avancement des projets lorsque le praticien passe de groupe en groupe. Pour les élèves, cette double contrainte semble interférer en partie avec l'avancement des projets, dans la mesure où, dans la plupart des groupes de trois ou quatre élèves, deux d'entre eux s'occupent presque uniquement des tâches d'archivage et de rédaction. Par ailleurs, d'autres tensions sont susceptibles d'émerger au cours de situations que les chercheurs ne sont pas susceptibles d'observer. En effet, la nécessité de rédiger, généralement chez soi, les retours à destination des élèves entre en concurrence avec les autres tâches qui incombent aux praticiens. Les groupes de discussion ont permis d'apporter un éclairage distinct sur ces données, confirmant le dépassement de certaines contradictions, mais aussi l'émergence de nouvelles.

\section{Les perspectives des enseignants sur la restructuration de leur activité}

Lors des discussions en groupe, les enseignants ayant utilisé le $C N E C$ dans une logique de rédaction incrémentale ont commenté la restructuration de leur activité, et souligné l'existence de diverses tensions dont ils estiment que le dépassement est lié à l'artefact utilisé. Déjà familiers avec la rédaction incrémentale avant le projet, ils soulignent l'intérêt d'une instrumentation numérique sur trois points : changement dans la régulation de l'activité des élèves, meilleure prise en compte des remarques faites sur les productions écrites, et évolution du rapport des élèves à l'erreur. 
Un dépassement partiel de certaines tensions grâce à l'application. La théorie de l'activité nous amène à nous centrer sur les contradictions qui semblent partiellement dépassées lors de l'utilisation des prototypes du CNEC. Les discussions de groupe confirment le fait que les praticiens favorisent désormais la rédaction de leurs retours en dehors du temps de classe, ce qui permet de constater un dépassement partiel de la contradiction secondaire entre les pôles « règles » et « instruments » identifiée précédemment. À la question « Faisiez-vous les commentaires écrits pendant le temps de classe ? », Isaline, exerçant à l'école primaire, déclare :

« Non, après. J'ai pas le temps. Quand il y a des protocoles expérimentaux qui se font en même temps, je dois avoir un œil dessus. Et justement ça me permet de pouvoir faire tout en même temps, parce que quand il y a de l'écrit, à partir de là les enfants peuvent bien se concentrer sur ce qu'ils ont à faire, et moi je peux travailler sur les expérimentations, le protocole, et ce qui se passe véritablement avec les gamins. »

Suivre une approche équivalente avec une instrumentation papier impliquerait qu'elle emporte chez elle l'ensemble des cahiers de la classe à chaque séance, ou qu'elle rédige ses retours pendant le temps qu'elle passe dans l'établissement. La pénibilité d'une telle démarche a fait consensus entre tous les enseignants du groupe de discussion.

Le deuxième constat réalisé est celui d'une meilleure prise en compte, par les élèves, des remarques de l'enseignant lorsque le CNEC est utilisé, ce qui, selon cette praticienne, limite le caractère répétitif des échanges qu'elle a avec de nombreux groupes d'élèves. Elle souligne les difficultés que rencontrent les élèves à prendre en compte les remarques faites oralement, car celles-ci sont facilement oubliées. Elle ajoute que l'écrit permet de formuler de manière plus construite ses commentaires :

« L'écrit permet justement pas d'oubli. Dans une classe il y a toujours du bruit quand on travaille comme ça en groupe, et là justement ça leur permet d'y aller progressivement et de revenir à la phase précédente s'ils n'ont toujours pas compris, progressivement, et ne pas oublier. Il y a une question d'oubli. Quand c'est à l'oral ils oublient la première partie de la phrase. Ils se souviennent plus en fin de compte quelle est la suite d'idées. Quand on est dans les commentaires on est souvent très logique et on essaie de les faire progresser à l'intérieur du 
commentaire, pour qu'ils arrivent à la fin à trouver la solution, et là justement

l'écrit est super aidant. »"

Une collègue, Victoire, rappelle néanmoins que si certaines tensions deviennent plus secondaires, d'autres apparaissent. Pour elle, ce passage par l'écrit constitue une charge de travail supplémentaire : «Par contre, c'est sûr que c'est plus de temps de travail pour moi à la maison », explique-t-elle. Armelle, enseignante de $\mathrm{SVT}^{2}$ au collège André Girault, rebondit sur ces propos en précisant : «Les collègues de primaire peuvent le faire parce qu'ils n'ont qu'une seule classe, mais moi jamais je pourrais faire ça avec mes neuf classes ». Pour souligner la distinction avec des retours écrits qui pourraient être réalisés sur papier, elle rappelle qu'il est possible de donner des devoirs à faire chez eux aux élèves de collège, contrairement aux élèves d'école primaire en France. Cela facilite la mise en place d'allers-retours avec ses élèves entre chaque séance et accélère ainsi l'amélioration des productions par rapport à une instrumentation papier.

Ces deux remarques invitent à faire la distinction entre les niveaux primaire et secondaire, les différences dans les conditions d'exercice des enseignants conduisant à l'apparition de tensions distinctes selon les contextes. Il y a néanmoins consensus sur l'évolution du rapport que les élèves entretiennent aux erreurs qu'ils commettent.

Une évolution du rapport à l'erreur. La contradiction secondaire liée à l'instrumentation de la rédaction incrémentale semble aussi évoluer au moment de l'utilisation du CNEC, car les élèves perçoivent moins les erreurs comme une sanction. Il faut alors distinguer, dans cette évolution, ce qui relève d'un changement de pratiques pédagogiques - le caractère incrémental de l'évaluation — et ce qui relève d'une instrumentation numérique. Pour ce faire, nous rapportons ci-après les propos de Matéo, enseignant de SVT exerçant au collège, puisqu'ils sont représentatifs des positions exprimées pendant la discussion de groupe. En ce qui concerne le changement de pratiques pédagogiques, il interprète la plus grande réceptivité des élèves face aux erreurs et à la nécessité de les corriger, comme une conséquence du caractère non définitif des retours de l'enseignant, qui ne sont pas associés à une évaluation finale de leur production.

2 Sciences de la vie et de la terre. 
«En fait tu es pas obligé de lui faire la réflexion de lui dire attention là reprend etc., devant les autres en fait. Parce que finalement tu vas juste lui dire vous ouvrez, vous allez sur la fiche-recherche, et vous regardez, vous reprenez ce qu'il $\mathrm{y}$ a à reprendre, et vous reprenez le travail à faire. En fait du coup, un élève qui va avoir tout vert, lui il va être content il va pouvoir continuer son travail, mais l'élève qui a tout jaune, finalement il va pas dire 'bah non j'ai pas envie de faire le travail" ou quoi que ce soit, ce qui peut arriver en classe quand on lui rend une copie et on lui dit toi t'as six, il faut corriger. »

Ce changement d'attitude est selon lui en partie lié à l'instrumentation numérique. Il est notamment plus difficile pour les élèves de comparer leur travail à celui de leurs pairs lorsque les retours sont faits sur un support numérique. Par opposition à des copies papier notées, où les élèves cherchent souvent à regarder les notes de leurs pairs, l'écran, à condition qu'il soit individuel, expose moins que la copie papier les erreurs et difficultés éventuelles d'un élève au reste de la classe.

« C'est surtout que du coup que le camarade qui a tout vert, il voit pas l'autre qui a tout jaune, [...]. S'il y en a où tu as tout coché en disant passe à la suite, ou l'autre tu as tout souligné, ou tout surligné en disant ça c'est à revoir ça c'est à revoir, en fait ils le voient parce que c'est sur la feuille. Là il y a la barrière de l'écran, qui fait qu'en fait ils sont concentrés sur leur écran à eux. »

Ainsi, le praticien défend que le rapport à l'évaluation formative se trouve modifié par le choix de l'instrumentation. Nous tâcherons, dans la section suivante, d'apporter des éléments pour interpréter cette position et reviendrons sur la manière dont la question de l'instrumentation numérique de la rédaction incrémentale renouvelle des questions anciennes.

\section{Discussion}

Par rapport aux recherches centrées sur les effets liés à la mise en place de rétroactions de la part des enseignants (Van de Pol et al., 2010) ou de leur instrumentation numérique (Vardi, 2012), nous nous sommes éloignés de la recherche de causalité associée à la question des gains de performance, qu'on la mesure sur le plan des caractéristiques des 
écrits ou des capacités d'autorégulation. Ce faisant, au regard des travaux menés sur la rédaction incrémentale, le principal apport de ce travail consiste à poser le regard sur les pratiques des enseignants et la manière dont ils restructurent leur activité pour mieux réguler leurs séances. Nous entamons la discussion de cet article en revenant sur les contradictions que l'instrumentation numérique de la rédaction incrémentale a contribué à dépasser. Nous poursuivons sur la question des limites de notre recherche, pour ouvrir sur des perspectives de recherches futures.

\section{Le dépassement des contradictions et l'évolution du système d'activité}

L'un des objectifs poursuivis à travers le développement de la fiche-recherche consistait à dépasser les contradictions au sein de l'activité des enseignants, entre le mandat du programme Savanturiers de développer la rédaction incrémentale et l'instrumentation papier mobilisée. Nous avons vu, pour l'échantillon d'enseignants suivis, que l'utilisation du $C N E C$ autorisait une meilleure régulation de l'activité en classe, permettant de consacrer davantage de temps aux groupes d'élèves peu investis, et offrant aux groupes plus actifs la possibilité de continuer à progresser dans leurs tâches sans avoir à attendre les retours de l'enseignant, puisqu'ils pourront être réalisés plus tard, de manière asynchrone.

Les observations en classe et les entretiens menés avec les praticiens corroborent l'hypothèse selon laquelle l'instrumentation numérique permet de dépasser certaines tensions dans l'activité des enseignants, en offrant notamment une meilleure régulation de celle-ci, et que cela rend l'instrumentation numérique plus acceptable par les praticiens. Cependant, l'introduction de l'artefact numérique entraîne également un surinvestissement en dehors du temps de classe et nous avons vu qu'une double instrumentation, numérique et papier, se mettait systématiquement en place. Il est dès lors probable que des contradictions tertiaires apparaissent dans de multiples contextes, entre les pôles « instruments » du nouveau et de l'ancien système d'activité, en plus des tensions inhérentes à l'utilisation d'appareils numériques en classe.

Les observations portent à croire que la gestion des problèmes liés à l'infrastructure technique (problèmes de connexion, de batterie, etc.) se double de tensions liées à un passage d'une rédaction individuelle à une rédaction collective. Ainsi, nous avons observé qu'au sein d'un même groupe les élèves se disputent régulièrement 
pour assumer le rôle de secrétaire afin d'être l'utilisateur de la tablette. Ce sont autant de points sur lesquels nous n'avons pas proposé d'analyse spécifique, puisqu'ils ne sont pas directement liés à la question de la rédaction incrémentale. Il est difficile à ce stade de déterminer dans quelle mesure l'émergence de telles tensions est susceptible de contrebalancer, aux yeux des enseignants, les bénéfices d'un dépassement d'anciennes contradictions via une instrumentation numérique.

Nos résultats sur le rapport à l'erreur des élèves permettent également d'envisager l'émergence d'une autre contradiction tertiaire concernant une évolution de l'objet de l'activité des enseignants. L'introduction dans la structure du CNEC d'une logique d'évaluation formative à travers le processus de rédaction incrémentale met les enseignants face à une évolution des approches évaluatives et les contraint, plus particulièrement, à mettre en œuvre une forme d'évaluation formative. Or, selon certains auteurs (Favre, 1995), cette approche de l'évaluation rencontre des réticences de la part des praticiens, indépendamment des questions d'instrumentation. Cette considération amène à revisiter des questions anciennes (Perrenoud, 2001) au prisme de technologies récentes, et notamment la question de l'évolution du rapport à l'erreur.

Dans une instrumentation de la rédaction incrémentale, l'artefact est pensé pour que les retours de l'enseignant aient pour fonction d'aider les élèves à améliorer leurs productions présentes, là où dans une évaluation notée les commentaires ne peuvent servir qu'à améliorer d'éventuelles productions futures. Il peut être utile de rappeler les réflexions de Favre (1995) sur l'enseignement scientifique et le statut des erreurs :

« La diffusion du concept d'évaluation formative ne s'est pas apparemment traduite par des modifications notables d'attitudes dans la façon d'enseigner. Cette conception "nouvelle" de l'évaluation attribue à l'erreur un statut et une signification très différents de ceux qu'elle a lorsqu'elle est confondue avec la notion traditionnelle de faute. » (p. 85)

Si l'on peut estimer que ces réticences jouent contre l'acceptabilité d'EIAH comme le $C N E C$, on peut également émettre l'hypothèse que l'introduction de nouveaux instruments numériques puisse faire évoluer cette tension propre à la représentation de l'erreur. Les propos de Matéo, rapportés précédemment, indiquent que cette hypothèse mérite d'être mise à l'épreuve. Néanmoins, à nouveau, la composition de notre échantillon limite toute forme de généralisation à ce stade, donnant à ce résultat le 
seul statut d'hypothèse à tester dans des travaux futurs, considération qui nous amène maintenant aux limites de la présente recherche.

\section{Les limites de la recherche}

La première limite de cette recherche correspond à la composition de l'échantillon d'enseignants suivis : nous avons fait face au compromis entre un nombre important de classes suivies et un nombre important de séances suivies par établissement. Dans la mesure où nous souhaitions réaliser un suivi longitudinal des projets, la seconde option a été préférée. Dès lors, nous n'avons pas été en mesure de contraster de nombreux contextes ; par ailleurs, nous travaillions avec des enseignants déjà engagés dans les projets Savanturiers et, ce faisant, ayant un regard plutôt positif vis-à-vis de la rédaction incrémentale. Enfin, il a été difficile de garder des enseignants engagés dans un même projet de recherche durant plusieurs années, aussi, seulement quatre praticiens ont pu être suivis à la fois avant et après l'introduction du CNEC. La possibilité de généraliser nos résultats s'en trouve donc affectée, notre recherche reste ainsi avant tout exploratoire.

La seconde limite de notre travail tient au fait que, pendant les groupes de discussion, les enseignants ont essentiellement évoqué l'introduction du CNEC en termes positifs. S'ils ont rappelé certaines difficultés rencontrées lorsque nous nous attardions sur la question, elles relevaient avant tout de problèmes techniques liés à l'artefact, encore au stade de prototype ${ }^{3}$, ou sur le manque de disponibilité pour mener le projet comme ils le souhaiteraient. Il est vraisemblable qu'un biais de désirabilité sociale (Nederhof, 1985), notamment lié au fait d'avoir participé au processus de conception, ait amené les enseignants sondés à minimiser certaines des difficultés constatées lors des observations.

\section{Conclusion}

En guise de perspective de recherche, nous proposons d'aborder la question des changements de pratiques enseignantes sous un angle nouveau, celui des traces d'apprentissage. Une autre spécificité des applications numériques comme le $C N E C$

3 Ces difficultés liées à des bogues techniques éphémères sont trop spécifiques d'une version intermédiaire de l'application pour être prises en compte dans les résultats et rapportées dans la présente contribution. 
réside dans le fait qu'il est possible de collecter des traces laissées par les utilisateurs au moment de leur manipulation de l'artefact. En s'inspirant de la méthodologie de travaux comme ceux de Faber et al. (2017), auteurs qui ont travaillé sur des outils d'évaluation formative, de futurs travaux pourraient viser à articuler des observations en classe et des analyses à grande échelle des utilisations de l'artefact, sans limitation du nombre d'enseignants pris en considération. Une telle approche permettrait de déterminer de manière plus précise sur quels créneaux horaires et pour quelles tâches les enseignants utilisent l'artefact afin de pallier les limites d'approches fondées sur des échantillons restreints. 


\section{Références}

Bonnat, C., Marzin-Janvier, P., Girault, I. et d'Ham, C. (2018). Modélisation didactique pour la conception d'étayages dans un EIAH : exemple d'une activité de conception expérimentale en biologie. STICEF, 25(2), 31-61. https://doi. org/10.3406/stice.2018.1766

Bourguin, G. (2000). Un support informatique à l'activité coopérative fondé sur la théorie de l'activité : le projet DARE [Thèse de doctorat, Université Lille 1]. https://www-lisic.univ-littoral.fr/publis/1394700486.pdf

Bourguin, G., Derycke, A. et Tarby, J. C. (2005). Systèmes interactifs en co-évolution : Réflexions sur les apports de la théorie de l'activité au support des pratiques collectives distribuées. Revue d'Interaction Homme-Machine, 6(1), 1-31. http://europiaproductions.free.fr/RIHM/V6N1/2-RIHM-Article\%20BourguinDerycke\%20PDF.pdf

Bywater, J. P., Chiu, J. L., Hong, J. et Sankaranarayanan, V. (2019). The teacher responding tool: Scaffolding the teacher practice of responding to student ideas in mathematics classrooms. Computers \& Education, 139(1), 16-30. https://www. learntechlib.org/p/209941/

Chan, C. K. K. et Chan, Y.-Y. (2011). Students' views of collaboration and online participation in knowledge forum. Computers \& Education, 57(1), 1445-1457. https://doi.org/10.1016/j.compedu.2010.09.003

Cisel, M. et Baron, G. L. (2019). Utilisation de tableaux de bord numériques pour l'évaluation des compétences scolaires : une étude de cas. Questions Vives, (31). https://doi.org/10.4000/questionsvives.3883

Cisel, M., Barbier, C. et Baron, G.-L. (2019). Rapport scientifique de synthèse de la recherche Cahier numérique de l'élève chercheur (CNEC) [Rapport de recherche]. Université Paris Descartes (Paris 5). https://hal.archives-ouvertes.fr/ $\underline{\text { hal- } 02278348}$

Coquidé, M., Fortin, C. et Rumelhard, G. (2009). L'investigation : fondements et démarches, intérêts et limites. ASTER, (49), 51-78. https://doi. org $/ 10.4267 / 2042 / 31129$ 
Dir, M. et Simonian, S. (2015). Analyse de l'activité tutorale dans un organisme privé de formation à distance selon le modèle d'Engeström : tensions et écarts au prescrit. Distances et Médiations des savoirs, (11). https://doi.org/10.4000/dms.1109

Edelson, D. C. (2001). Learning-for-use: A framework for the design of technology-supported inquiry activities. Journal of Research in Science teaching, 38(3), 355-385. https://doi. org/10.1002/1098-2736(200103)38:3\%3C355::AID-TEA1010\%3E3.0.CO;2-M

Engeström, Y. (1987). Learning by expanding. Orienta-Konsultit Oy.

Engeström, Y. (2000). Activity theory as a framework for analyzing and redesigning work. Ergonomics, 43(7), 960-974. https://doi.org/10.1080/001401300409143

Engeström, Y. (2008). Weaving the texture of school change. Journal of Educational Change, 9(4), 379-383. https://doi.org/10.1007/s10833-008-9086-6

Engeström, Y. (2011). Théorie de l'activité et management. Management et Avenir, 42(2), 170-182. https://doi.org/10.3917/mav.042.0170

Faber, J. M., Luyten, H. et Visscher, A. J. (2017). The effects of a digital formative assessment tool on mathematics achievement and student motivation: Results of a randomized experiment. Computers \& Education, 106, 83-96. https://doi. org/10.1016/j.compedu.2016.12.001

Favre, D. (1995). Conception de l'erreur et rupture épistémologique. Revue française de pédagogie, (111), 85-94. https://doi.org/10.3406/rfp.1995.1234

Frascara, J. (dir.). (2002). Design and the social sciences: Making connections. CRC Press.

Hattie, J. et Timperley, H. (2007). The power of feedback. Review of Educational Research, 77(1), 81-112. https://doi.org/10.3102/003465430298487

Hong, H.-Y., Scardamalia, M., Messina, R. et Teo, C. L. (2015). Fostering sustained idea improvement with principle-based knowledge building analytic tools. Computers \& Education, 89, 91-102. https://doi.org/10.1016/j.compedu.2015.08.012

Impedovo, M. A. et Andreucci, C. (2016). Co-élaboration de connaissances nouvelles : du modèle théorique à ses outils technologiques. STICEF, 23(2), 57-74. https://doi. org/10.3406/stice.2016.1702 
Jamet, E. (2006). Une présentation des principales méthodes d'évaluation des EIAH en psychologie cognitive. STICEF, 13, 129-146. https://doi.org/10.3406/ $\underline{\text { stice. } 2006.929}$

Kluger, A. N. et DeNisi, A. (1996). The effects of feedback interventions on performance: A historical review, a meta-analysis, and a preliminary feedback intervention theory. Psychological Bulletin, 119(2), 254-284. https://doi. org/10.1037/0033-2909.119.2.254

Krueger, R. A. (2014). Focus groups: A practical guide for applied research. SAGE.

Laferrière, T. et Lamon, M. (2010). Knowledge Building/Knowledge Forum: The transformation of classroom discourse. Dans M. S. Khine et I. M. Saleh (dir.), New science of learning: Cognition, computers, and collaboration (p. 485-502). Springer-Verlag.

Linn, M. C., Davis, E. A. et Bell, P. (dir.). (2013). Internet environments for science education. Routledge.

Nederhof, A. J. (1985). Methods of coping with social desirability bias: A review. European Journal of Social Psychology, 15(3), 263-280. https://doi.org/10.1002/ ejsp.2420150303

Nielsen, J. (1993). Usability engineering. Morgan Kaufmann.

Nogry, S., Jean-Daubias, S. et Ollagnier-Beldame, M. (2004). Évaluation des EIAH : une nécessaire diversité des méthodes. Dans C. Frasson, F. Peccoud et C. Moreau (resp.), TICE 2004 : actes du colloque, Université de Technologie de Compiègne, les 21, 22 et 23 octobre 2004 (p. 265-271). Université de Technologie de Compiègne. https://edutice.archives-ouvertes.fr/edutice-00000729/document

Pedaste, M., Mäeots, M., Siiman, L., de Jong, T., van Riesen, S. A. N., Kamp, E. T., Manoli, C. C., Zacharia, Z. C. et Tsourlidaki, E. (2015). Phases of inquiry-based learning: Definitions and the inquiry cycle. Educational Research Review, 14, 47-61. https://doi.org/10.1016/j.edurev.2015.02.003

Perrenoud, P. (2001). Évaluation formative et évaluation certificative : postures contradictoires ou complémentaires? Formation professionnelle suisse, (4), 25-28. http://www.unige.ch/fapse/SSE/teachers/perrenoud/php_main/ php_2001/2001_13.html 
Quintana, C., Reiser, B. J., Davis, E. A., Krajcik, J., Fretz, E., Duncan, R. G., Kyza, E., Edelson, D. et Soloway, E. (2004). A scaffolding design framework for software to support science inquiry. Journal of the Learning Sciences, 13(3), 337-386. https://doi.org/10.1207/s15327809j1s1303_4

Sandoval, W. A. (2004). Developing learning theory by refining conjectures embodied in educational designs. Educational Psychologist, 39(4), 213-223. https://doi. org/10.1207/s15326985ep3904_3

Scardamalia, M. (2004). CSILE/Knowledge forum ${ }^{\circledR}$. Dans A. Kovalchick et K. Dawson (dir.), Education and technology: An encyclopedia (p. 183-192). ABC-CLIO.

Scardamalia, M. et Bereiter, C. (2006). Knowledge building: Theory, pedagogy, and technology. Dans K. Sawyer (dir.), Cambridge handbook of the learning sciences (p. 97-118). Cambridge University Press.

Slotta, J. D. et Linn, M. C. (2009). WISE Science: Web-based inquiry in the classroom. Teachers College Press.

Tabak, I. et Reiser, B. J. (1997). Complementary roles of software-based scaffolding and teacher-student interactions in inquiry learning. Dans CSCL' 97 : Proceedings of the 2nd international conference on Computer support for collaborative learning, December 10-14, 1997, Toronto, Canada (p. 292-301). https://doi. org/10.3115/1599773.1599809

Tricot, A., Plégat-Soutjis, F., Camps, J.-F., Amiel, A., Lutz, G. et Morcillo, A. (2003). Utilité, utilisabilité, acceptabilité : interpréter les relations entre trois dimensions de l'évaluation des EIAH. Dans C. Desmoulins, P. Marquet, D. Bouhineau (dir.), Environnements informatiques pour l'apprentissage humain (p. 391-402). ATIEF/ INRP. https://edutice.archives-ouvertes.fr/edutice-00000154/document

Van de Pol, J., Volman, M. et Beishuizen, J. (2010). Scaffolding in teacher-student interaction: A decade of research. Educational Psychology Review, 22(3), 271296. https://doi.org/10.1007/s10648-010-9127-6

Vardi, I. (2012). The impact of iterative writing and feedback on the characteristics of tertiary students' written texts. Teaching in higher education, 17(2), 167-179. https://doi.org/10.1080/13562517.2011.611865 
Zhao, K. et Chan, C. K. K. (2014). Fostering collective and individual learning through knowledge building. International Journal of Computer-Supported Collaborative Learning, 9(1), 63-95. https://doi.org/10.1007/s11412-013-9188-x 